\title{
Antagonistic characteristics are positively associated with inflammatory markers independently of trait negative emotionality
}

\author{
Anna L. Marsland ${ }^{\mathrm{a}, *}$, Aric A. Prather ${ }^{\mathrm{a}}$, Karen L. Petersen ${ }^{\mathrm{b}}$, Sheldon Cohen ${ }^{\mathrm{c}}$, \\ Stephen B. Manuck ${ }^{\mathrm{d}}$ \\ a Behavioral Immunology Laboratory, Department of Psychology, University of Pittsburgh, 3943 O'Hara Street, Pittsburgh, PA 15260, USA \\ ${ }^{\mathrm{b}}$ Minneapolis Veterans Administration Medical Center, Minneapolis, MN 55417, USA \\ ${ }^{c}$ Department of Psychology, Carnegie Mellon University, Pittsburgh, PA 15213, USA \\ ${ }^{\mathrm{d}}$ Behavioral Physiology Laboratory, Department of Psychology, University of Pittsburgh, Pittsburgh, PA 15260, USA
}

Received 24 September 2007; received in revised form 26 November 2007; accepted 27 November 2007

\begin{abstract}
Recent evidence suggests that individuals with certain personality traits are at elevated risk for chronic systemic inflammation. To date, this literature has focused on the related traits of hostility and negative affect (NA). In this study, we examine the covariation of trait measures of hostility and NA with the inflammatory mediators interleukin-6 and C-reactive protein. We also explore whether observed associations reflect independent contributions of cognitive, affective and behavioral components of hostile dispositions or shared trait variance with global negative affectivity. Subjects were a diverse sample of 855 relatively healthy middle-aged community volunteers (50\% male) from the Adult Health and Behavior Project. The Buss and Perry Aggression Questionnaire (BPAQ) and an Abbreviated Cook-Medley Hostility Scale (ACM) were used to measure dimensions of hostility, and the Multidimensional Personality Questionnaire was used to measure trait NA. Regression analyses accounting for demographic characteristics and medical covariates showed a positive relationship of all components of hostility and trait NA with both IL-6 and CRP. After controlling for trait NA, only the behavioral component of hostility was independently associated with the inflammatory markers. The relationships of cognitive and affective components of hostility with inflammatory markers were largely explained by lifestyle factors, particularly body mass index and smoking. In contrast, lifestyle factors did not explain the covariation of hostile behavioral tendencies and inflammation. These findings suggest that unique attributes of aggressive behavioral tendencies account for much of the variability in inflammation associated with hostility and negative emotions, raising the possibility that individuals high in aggression are at increased risk of inflammatory disease. (C) 2007 Elsevier Inc. All rights reserved.
\end{abstract}

Keywords: Personality; Hostility; Negative affect; Aggression; Interleukin-6; C-reactive protein; Inflammation

\section{Introduction}

There is abundant evidence that chronic elevation of systemic inflammation plays a role in the pathogenesis and course of numerous age-related conditions, including diabetes, cardiovascular disease, arthritis, and general functional decline (e.g., Black, 2003; Ferrucci et al., 1999; Pradhan et al., 2001, 2002; Ridker et al., 2000a,b). Indeed,

\footnotetext{
* Corresponding author. Fax: +1 4126483720.

E-mail address: marsland@pitt.edu (A.L. Marsland).
}

epidemiologic studies show that higher levels of proinflammatory mediators, such as interleukin (IL)-6 or C-reactive protein (CRP), predict future morbidity among initially healthy mid-life individuals (e.g., Libby and Ridker, 1999). Mounting evidence also suggests that individuals with certain personality traits are at elevated risk for adverse health outcomes, and that inflammatory mechanisms may underlie the pathogenicity of these dispositional attributes (Black, 2003; Suarez, 2004).

To date, studies examining personality-related correlates of systemic inflammation have focused on the traits of anger and hostility. For example, Ranjit et al. (2007) 
recently showed a positive relationship between hostility, measured using the cynical distrust subscale of the CookMedley Hostility (Ho) Scale (Cook and Medley, 1954), and circulating levels of CRP and IL-6 among 6814 healthy men and women aged 45-84 years. Recent smaller studies also show a positive association between CRP and trait anger/hostility (Coccaro, 2006; Graham et al., 2006; Suarez, 2004). For example, Suarez (2004) administered the Buss-Perry Aggression Questionnaire (BPAQ; Buss and Perry, 1992) to 127 healthy, young adults and showed that CRP covaried positively with scores on the anger subscale, but was not associated with the hostility subscale. Similarly, in a sample of older adults, Graham et al. (2006) showed a positive association between hostility measured with Ho scale and CRP that was independent of depressive symptomatology. Findings from smaller studies examining IL-6 are more mixed. To date, these studies have used the Ho scale and IL-6 has been shown to covary positively with the cynicism and hostile affect Ho subscales in one study of mid-life adults (Sjogren et al., 2006), but not with the total Ho score in younger (Suarez, 2003) or older (Graham et al., 2006) samples. In a sample of 100 men and women (50\% with clinical depression), Miller et al. (2003) found no main effect of Ho cynical hostility; however, among individuals scoring low in depressive symptoms, hostility was positively associated with IL-6. Further support for an interaction between hostility and depressive symptomatology in the prediction of IL-6 comes from a study of 90 healthy, young men. Here, in contrast to Miller et al.'s (2003) findings, Ho score was positively related to IL-6 only among men with higher depressive symptoms (Suarez et al., 2003).

Inconsistencies across studies may reflect heterogeneity of the anger and hostility measures, with results varying as a function of different affective, attitudinal, and expressive elements. Alternatively, it may be noted that anger and hostility share common variance with other negatively valenced dimensions of emotion, such as depression and anxiety, and that while phenomenologically distinct, these affective traits tend to cohere psychometrically under a common factor of neuroticism, or negative affectivity (NA) (Costa and McCrae, 1985; Suls and Bunde, 2005; Watson, 2000; Watson and Tellegen, 1985). It might be asked, then, whether the inflammatory cytokines are associated principally with the unique attributes of anger and hostility (e.g., their attitudinal or expressive quality) or with a dimensional feature of these traits that is common to other negative emotions, such as NA. In this regard, the goal of the current study was to examine covariation of the inflammatory mediators IL-6 and CRP with cognitive, affective and behavioral components of hostility, and to determine whether any observed associations reflect independent contributions of these antagonistic dimensions or shared trait variance in negatively valenced affect.

Another issue that requires clarification in the literature linking dispositional characteristics to inflammation is the role of health practices. Trait measures of negative affectiv- ity (NA) have been associated with lifestyle risk factors, including smoking, poor diet, sedentary lifestyle, obesity, and alcoholism (e.g., Everson et al., 1997; Kawachi et al., 1996). Furthermore, these same lifestyle factors covary positively with levels of peripheral IL-6 and CRP (Kiecolt-Glaser and Glaser, 1988; Khaodhiar et al., 2004). In particular, evidence shows that adipocytes produce 10 $35 \%$ of circulating IL-6 (Berg and Scherer, 2005; Mohamed-Ali et al., 1997), which, in turn, stimulates the peripheral production of CRP by hepatocytes (Heinrich et al., 1990). Thus, the higher levels of body fat or poorer health practices that accompany trait NA could explain concomitant systemic inflammation. To explore this possibility, a secondary goal of the current study was to examine whether any observed associations of inflammatory mediators with dimensions of hostility and NA are accounted for by body mass index (BMI), current smoking, sleep volume, physical activity, and/or alcohol use.

\section{Methods}

\subsection{Participants}

Data for the present study were derived from the University of Pittsburgh Adult Health and Behavior (AHAB) project, a registry of behavioral and biological measurements on non-Hispanic Caucasian and African American individuals (30-54 years old) recruited via mass-mail solicitation from communities of southwestern Pennsylvania, USA (principally Allegheny County). Exclusion criterion for entry into the AHAB study included a reported history of atherosclerotic cardiovascular disease, chronic kidney or liver disease, cancer treatment in the preceding year, and major neurological disorders, schizophrenia or other psychotic illness. Other exclusions included pregnancy and the use of insulin, glucocorticoid, anti-arrhythmic, psychotropic, or prescription weight-loss medications. Data collection occurred over multiple laboratory sessions, and informed consent was obtained in accordance with approved protocol guidelines of the University of Pittsburgh Institutional Review Board.

For the current analyses, we identified 1081 AHAB participants on whom we had measures of plasma IL-6 and CRP. Given that absence of common acute illnesses (e.g., recent colds or allergies) was not an exclusionary criterion for the AHAB study, we dropped any individuals with IL-6 levels greater than $10 \mathrm{pg} / \mathrm{ml}$ or CRP levels greater than $10 \mathrm{mg} / \mathrm{L}$ $(n=142)$ from the current sample. In addition, we excluded individuals with rheumatoid arthritis $(n=1)$, diabetes $(n=15)$, or a history of chronic hepatitis $\mathrm{B}$ or $\mathrm{C}(n=3)$, and those taking antibiotics $(n=23)$, cold medications $(n=33)$, immunosuppressants $(n=3)$, or inhaled corticosteroids $(n=31)$, resulting in a final sample of 855 subjects.

\subsection{Procedure}

Participants in the AHAB project visited the laboratory for four individually-scheduled sessions. Data obtained at sessions 1 and 2 are included here (on average these sessions were less than 2 weeks apart). At session 1 , written informed consent was obtained and participants completed a battery of computer-administered demographic, psychological and health behavior measures. Blood was drawn for the determination of plasma levels of IL-6 and CRP at session 2, which was scheduled in the morning. Prior to coming into the laboratory for this session, participants were asked to fast for $8 \mathrm{~h}$ and avoid exercise for $12 \mathrm{~h}$ and alcohol for $24 \mathrm{~h}$. On arrival, a project nurse administered a medical history interview, including questions about past history of rheumatoid or osteoarthritis, diabetes, chronic hepatitis $\mathrm{B}$ or $\mathrm{C}$, acute kidney disease, cancer, asthma 
or other lung conditions, and thyroid disease, and measured several standard risk factors, including blood pressure and BMI $\left(\mathrm{kg} / \mathrm{m}^{2}\right)$, and drew a $40 \mathrm{cc}$ blood sample.

\subsection{Measures}

Personality: Trait anger/hostility was measured using two scales: (1) an abbreviated 39-item version of the Cook-Medley Hostility Scale (Ho; Cook and Medley, 1954), as derived by Barefoot et al. (1995) and (2) the 29-item Buss and Perry Aggression Questionnaire (BPAQ; Buss and Perry, 1992). Following Boyle et al. (2007), we examined items from the Cook-Medley Hostility Scale that reflect the cognitive (cynicism and hostile attributions subscales), affective (hostile affect subscale), and behavioral (aggressive responding subscale) components of hostility. Testretest reliability for the AHo was .74 over a 10-year period (Barefoot, 1997). The Cook-Medley Hostility Scale has also been shown to have good internal consistency (Contrada and Jussim, 1992) and construct validity (Smith and Frohm, 1985). The BPAQ also includes subscales that reflect the cognitive (hostility), affective (anger), and behavioral (physical aggression and verbal aggression) components of antagonistic dispositions. This measure has good reliability (test-retest reliability $=0.80$ ) and internal consistency (Cronbach's alphas $=.72-.85$ for subscales and total score; Buss and Perry, 1992). To yield a single measure of the cognitive, affective and behavioral dimensions of hostility, we combined parallel subscales from the AHo and BPAQ by averaging standardized scores $(r$ 's for similar scales from the 2 instruments ranged from .45 to .63). To measure trait NA, participants also completed the Multidimensional Personality Questionnaire-Short Form (MPQ-SF; Tellegen, 1982), a 155 item measure of broad temperament domains including positive emotionality, negative emotionality, and constraint.

Plasma IL-6 and CRP: Plasma samples for the determination of IL-6 and CRP were collected in citrated plasma tubes and frozen at $-80^{\circ} \mathrm{C}$ until analysis in batches. IL-6 levels were determined using a high sensitivity quantitative sandwich enzyme immunoassay kit (R\&D Systems). Briefly, standards, controls and samples were added to a 96-well microplate pre-coated with monoclonal anti-IL-6 antibodies. Unbound substances were removed by washing and an enzyme linked polyclonal antiIL-6 antibody was then added. This was followed by washing, incubation with a substrate solution and then with the amplifier solution. The intensity of the color that resulted was measured at $490 \mathrm{~nm}$. The assay standard range is $0.156-10 \mathrm{pg} / \mathrm{mL}$. IL-6 levels were extrapolated from a standard curve with linear regression from a log-linear curve. All samples were run in duplicate and the average coefficient of variation between samples was $5 \%$. Reciprocal transformation was applied to normalize raw score distributions of the IL-6 values. For ease of interpretation, the signs of reciprocally transformed measurements of IL- 6 were then reversed.

CRP was measured at the University of Vermont's Laboratory of Clinical Biochemistry Research with the BNII nephelometer from Dade Behring utilizing a particle enhanced immunonephelometric assay. In this procedure, polystyrene particles are coated with monoclonal antibodies to CRP, which, in the presence of antigen agglutinate cause an increase in the intensity of scattered light. The increase in scattered light is proportional to the amount of CRP in the sample. The assay range is 0.175 $1100 \mathrm{mg} / \mathrm{L}$. Intra-assay CVs range from 2.3 to $4.4 \%$ and inter-assay CVs range from 2.1 to $5.7 \%$. Final CRP values were log normal (base e) transformed prior to analysis to better approximate a normal distribution.

\subsection{Covariates}

All analyses included a group of standard covariates, including age, gender, race, and years of education. Medical conditions and current medications, as reported by participants in the medical history interview, were also employed as standard covariates if they were associated with the inflammatory markers. Secondary analyses also examined a number of lifestyle factors that might explain associations between trait characteristics and inflammation. These included BMI, smoking status (current smoker versus ex/non-smoker), sleep volume (hours of sleep during last 7 nights $=($ average hours/week night $\times 5)+($ average hours/weekend night $\times 2$ ), physical activity (estimated kilocalories expended per week by the Paffenbarger Physical Activity Questionnaire; Paffenbarger et al., 1993), and alcohol use (average number of alcoholic drinks/week). Scores for BMI, alcoholic drinks/week, and physical activity were log normal (base e) transformed prior to analysis.

\subsection{Statistical analyses}

All analyses were conducted using SPSS version 15.0 (SPSS Inc., Chicago, IL.). Initially, a series of Pearson product-moment and point biserial correlations were calculated to examine relationships between IL-6, CRP and demographic characteristics, health behaviors and psychological traits. Next, linear regression analyses were used to determine the variance in IL-6 and CRP levels accounted for by the dimensions of hostility and NA. For the initial analyses, age, gender, race, years of education and identified medical covariates were entered in the first step of the model, followed by the trait characteristic of interest. Secondary analyses were then conducted to examine whether the cognitive, affective, and behavioral dimensions of hostility were associated with IL- 6 and CRP independently of NA. Here, demographic and medical covariates were entered in step 1, NA in step 2, and hostility scores in step 3 of three separate regression models. Finally, similar hierarchical techniques were employed to examine whether negative dispositions predicted inflammatory indices independently of BMI and health practices.

\section{Results}

\subsection{Associations between demographic and health characteristics and inflammatory markers}

Demographic characteristics of the sample and bivariate correlations between inflammatory markers and demographic and health risk factors are presented in Table 1. Higher levels of peripheral IL-6 and CRP were associated with fewer years of education, African American race, less physical activity, and higher BMI. In addition, plasma IL6 , but not CRP, was positively associated with smoking and fewer hours of sleep. In sum, these findings show that demographic and health-related risk factors are generally associated with higher values for markers of systemic inflammation.

In regard to past medical history, there were no associations between inflammatory markers and self-reported history of acute kidney disease $(n=39)$, cancer $(n=19)$, asthma $(n=43)$, other lung conditions $(n=46)$, or thyroid disease $(n=40)$. History of osteoarthritis $(n=109)$ was associated with higher levels of both IL-6 and CRP $(t(1,853)=-2.33, \quad p=.02 ; \quad t(1,853)=-2.46, \quad p=.01$, respectively). Hypertension, recorded as a resting blood pressure above $130 / 85$ or use of anti-hypertensive medications (diuretics $(n=20)$, beta blockers $(n=12)$, calcium blockers $(n=11)$, central anti-hypertensives $(n=1)$, and/ or ace inhibitors $(n=25))$ was also associated with higher circulating levels of IL-6 and CRP $(t(1,853)=-4.94$; $p<.001 ; \quad t(1,853)=-5.49 ; \quad p<.001, \quad$ respectively). In regard to other medications, there were no associations between IL-6 or CRP and current use of anti-lipenics $(n=22)$ or antacids $(n=11)$. Higher levels of CRP were associated with hormone replacement treatment (HRT: 


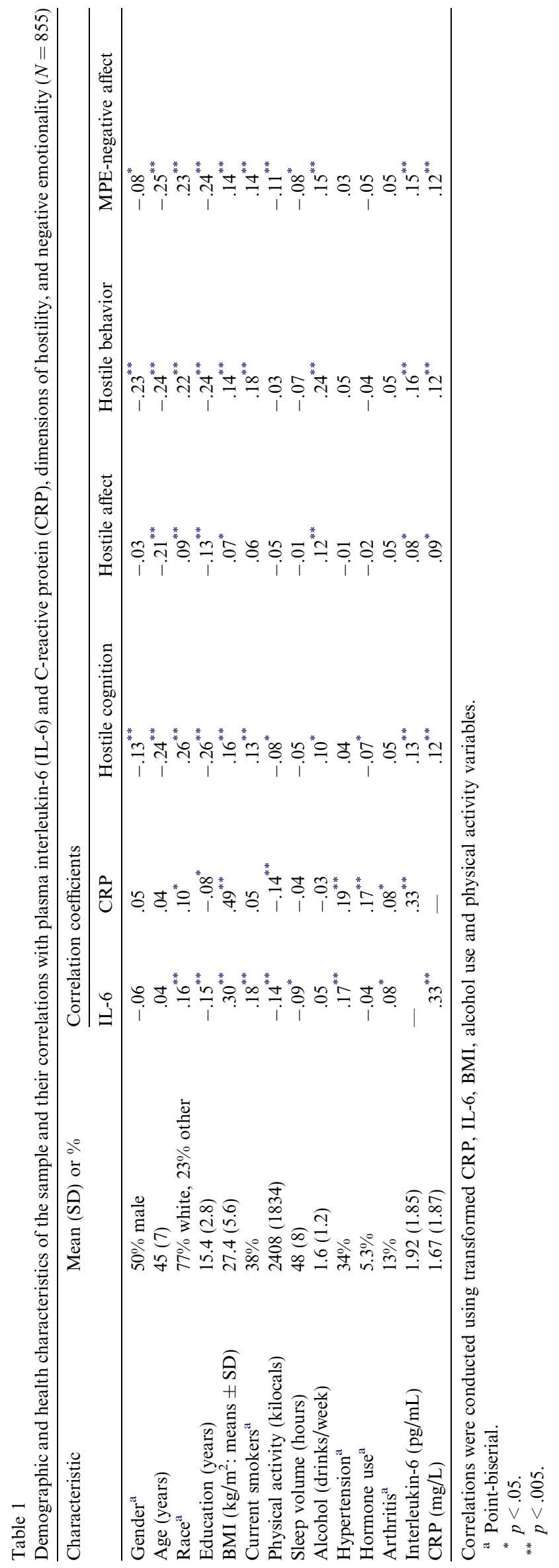

$n=19 ; t(1,853)=-2.93 ; p=.003)$ and use of oral contraceptives $(n=26 ; t(1,853)=-4.18 ; p<.001)$. No participants reported taking nitrates, anti-arrhythmics, oral hypoglycemics, protease inhibitors, or anti-HIV medications. Based on these findings, history of osteoarthritis (by self report) and hypertension (blood pressure $>130 / 85$ or use of anti-hypertensive medications) were included as covariates in all models, and hormone use (HRT or oral contraceptives) on analysis of CRP.

\subsection{Associations between dispositional characteristics and inflammation}

Next, we assessed whether psychological traits were associated with circulating levels of IL-6 and CRP. Results of preliminary bivariate analyses are presented in Table 1. Both markers of systemic inflammation were positively associated with cognitive, affective, and behavioral components of hostility, and trait NA. There were no significant relationships between inflammation and the MPQ-SF dimensions of positive emotionality or constraint. In light of these initial findings, we went on to conduct a series of multiple regression analyses assessing associations between trait negative characteristics and IL-6 and CRP after controlling for standard demographic covariates, history of osteoarthritis, and current hypertension, in all models, and hormone use on analysis of CRP. Results of these analyses are presented in Table 2 . After entering the covariates, regression analyses revealed a positive relationship of cognitive, affective and behavioral components of hostility and NA with CRP. Similarly, hostile cognitions and behaviors and NA covaried positively with IL-6, with a trend on analysis of hostile affect. Further regression analyses showed no significant interactions between any of the hostility components and NA or between demographic (gender, age, race, or years of education) or health-related risk factors (history of arthritis, hypertension or hormone use) and dispositional characteristics in predicting markers of inflammation. Fig. 1 summarizes the association between the inflammatory mediators and the behavioral component of hostility. To simplify the presentation, levels of the trait characteristic were categorized by quartiles for this figure (Group $1(n=213)$ : mean hostile behavior score $=-.91(S D=.22)$; Group $2(n=214)$ : mean $=-.36$ $(S D=.14)$; Group $3(n=214)$ : mean $=.17(\mathrm{SD}=.19)$; Group $4(n=213)$ : mean $=1.10(\mathrm{SD}=.79))$.

\subsection{Relationships between the dispositional constructs in the prediction of inflammation}

To examine whether hostile characteristics and NA reflect the same underlying dispositional construct, we first examined Pearson product-moment correlations between these psychological characteristics. As expected, NA was closely related to hostile cognitions $(r=.69, p<.0001)$, affect $(r=.60, \quad p<.0001)$, and behavior $(r=.56$, $p<.0001)$. Thus, we ran three regression analyses, entering 
Table 2

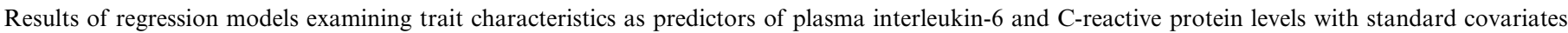
entered in step 1 and psychological traits entered into step 2 of separate models $(n=855)$

\begin{tabular}{|c|c|c|c|c|c|c|}
\hline \multirow[t]{2}{*}{ Predictor } & \multicolumn{3}{|c|}{ Transformed IL-6 } & \multicolumn{3}{|c|}{ Transformed CRP } \\
\hline & Beta & $p$-Value & $\operatorname{Adj} R^{2}$ & Beta & $p$-Value & $\operatorname{Adj} R^{2}$ \\
\hline Step 1 & & & .066 & & & .084 \\
\hline Gender & -.06 & .09 & & .06 & .08 & \\
\hline Age & .03 & .47 & & -.07 & .04 & \\
\hline Race & .13 & .001 & & .05 & .15 & \\
\hline Education & -.11 & .002 & & -.04 & .25 & \\
\hline Arthritis & .06 & .07 & & .07 & .03 & \\
\hline Hypertension & .12 & .001 & & .19 & .001 & \\
\hline Hormone use & - & - & & .16 & .001 & \\
\hline \multicolumn{7}{|c|}{ Step 2 of separate models } \\
\hline Hostile cognitions $^{\mathrm{a}}$ & .07 & .05 & .070 & .11 & .002 & .094 \\
\hline Hostile affect ${ }^{\mathrm{a}}$ & .06 & .06 & .070 & .07 & .03 & .089 \\
\hline Hostile behavior $^{\mathrm{a}}$ & .12 & .002 & .077 & .11 & .003 & .094 \\
\hline $\begin{array}{l}\text { MPQ-negative } \\
\text { Emotionality }\end{array}$ & .10 & .006 & .072 & .10 & .007 & .092 \\
\hline
\end{tabular}

a Average standardized scores on the subscales of the Cook-Medley Hostility Scale and the Buss-Perry Aggression Questionnaire that measure cognitive, affective, and behavioral dimensions of hostility.

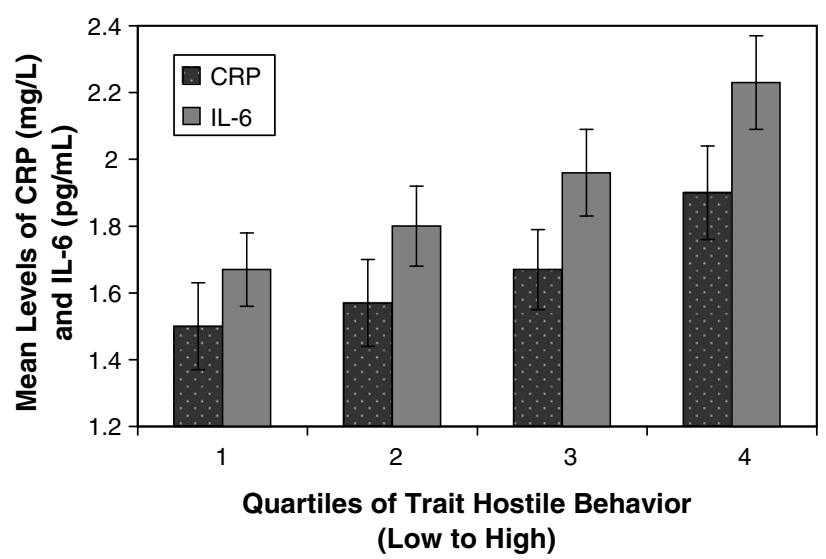

Fig. 1. Untransformed mean values of C-reactive protein and interleukin6 among individuals in the hostile behavior quartile groups (Group 1 $(n=213)$ : mean hostile behavior score $=-.91 \quad(S D=.22)$; Group 2 $(n=214):$ mean $=-.36 \quad(S D=.14) ;$ Group $3 \quad(n=214):$ mean $=.17$ $(S D=.19)$; Group $4(n=213)$ : mean $=1.10(S D=.79)$.

NA after the standard controls in a second step and each dimension of hostility in a third step of separate models. Results of these analyses are presented in Table 3. With NA in the model, there was an independent relationship between the behavioral dimensions of hostility and circulating levels of IL-6 $(b=.09, p=.04)$ and CRP $(b=.08$, $p=.04)$. The positive relationship between hostile cognitions and CRP was also partially retained $(b=.09$, $p=.057)$. In contrast, there were no significant associations between hostile cognitions and IL-6 or hostile affect and IL-6 or CRP with NA in the model. These findings suggest that relationships of affective dimensions of hostility with inflammatory markers are largely attributable to correlated variance in trait NA. A similar pattern is seen for cognitive dimensions of hostility, with trait NA partially accounting for observed relationships. In contrast, the behavioral component of hostility shows an association with inflammation that is independent of dispositional NA. Finally, when trait NA was entered into a third step of a model that included all dimensions of hostility, NA was not independently associated with levels of CRP $(p=.54)$ or IL-6 ( $b=.07, p=.14)$, suggesting that NA did not contribute anything unique to the prediction of systemic inflammation over correlated variation in hostility.

\subsection{The role of health practices}

Further, zero-order correlations revealed that the components of hostility were associated with a number of lifestyle factors, including BMI, smoking, alcohol use, and physical activity (See Table 1). Given that all of these factors, except alcohol use, were also associated with peripheral IL-6 and CRP, we next examined whether these lifestyle factors might account for associations between antagonistic characteristics and inflammatory markers. For this purpose, we entered BMI, smoking, and physical activity into the second step, and the trait characteristics into a third step of separate regression equations with the standard demographic and medical covariates already in the model. Entering health behaviors into the model reduced the magnitude of observed associations between hostile behavioral tendencies and circulating IL-6 (beta changed from .12 to .08 ) and CRP (beta changed from .11 to .06); however, hostile behavioral tendencies retained an independent association with both inflammatory mediators $(p$ 's $=.03$ and .05 , respectively) (See Table 4). Similarly, there was a trend for a relationship between hostile cognitions and CRP with health factors in the model $(b=.06, p=.06)$. In contrast, there were no significant associations between hostile cognitions and IL-6 or between hostile or negative affect and either inflammatory mediator with health behaviors in 
Table 3

Results of regression models examining relationships of hostility dimensions with plasma interleukin-6 and C-reactive protein after controlling for trait NA

\begin{tabular}{|c|c|c|c|c|c|c|}
\hline \multirow[t]{2}{*}{ Predictor } & \multicolumn{3}{|c|}{ Transformed IL-6 } & \multicolumn{3}{|c|}{ Transformed CRP } \\
\hline & Beta & $p$-Value & Adj $R^{2}$ & Beta & $p$-Value & $\operatorname{Adj} R^{2}$ \\
\hline \multicolumn{7}{|l|}{ Step 1} \\
\hline \multicolumn{7}{|l|}{ Step 2} \\
\hline Trait negative affect & .10 & .007 & .072 & .094 & .009 & .092 \\
\hline \multicolumn{7}{|l|}{ Step 3 of separate models } \\
\hline Hostile behavior $^{\mathrm{a}}$ & .088 & .039 & .077 & .084 & .043 & .096 \\
\hline
\end{tabular}

For these analyses, standard demographic (gender, age, race, education) and health (arthritis, hypertension, and hormone use) factors were entered in step

1 , trait negative affect in step 2, and the cognitive, affective, or behavioral dimension of hostility in step 3 of Separate Models $(n=855)$.

a Average standardized scores on the subscales of the Cook-Medley Hostility Scale and the Buss-Perry Aggression Questionnaire that measure cognitive, affective, and behavioral dimensions of hostility.

Table 4

Results of regression models examining relationships of hostility dimensions with plasma interleukin-6 and C-reactive protein after controlling for lifestyle factors

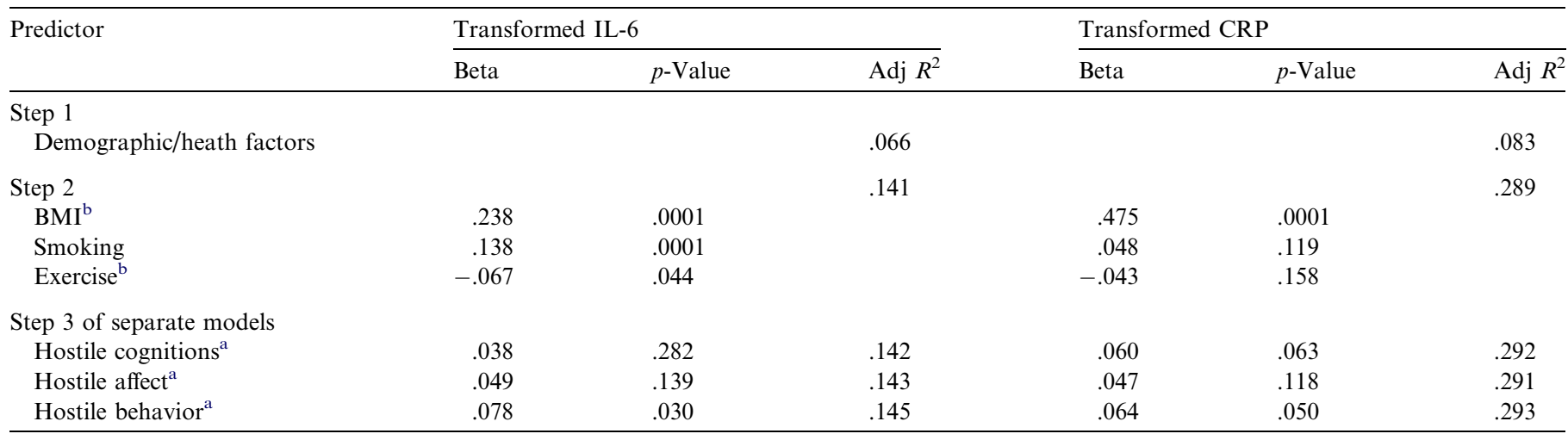

For these analyses, standard demographic (gender, age, race, education) and health (arthritis, hypertension, and hormone use) factors were entered in step 1 , body mass index, smoking status, and physical activity in step 2, and the cognitive, affective, or behavioral dimension of hostility in step 3 of Separate Models $(n=855)$.

a Average standardized scores on the subscales of the Cook-Medley Hostility Scale and the Buss-Perry Aggression Questionnaire that measure cognitive, affective, and behavioral dimensions of hostility.

b Scores were log normal (base e) transformed prior to analysis.

the model. Thus, these results suggest that lifestyle factors (in particular BMI and smoking) account for much of the variance in inflammation associated with hostile cognitions and affect.

\section{Discussion}

The present study provides initial evidence for an association between antagonistic behavioral tendencies and higher levels of systemic inflammation in a relatively healthy, mid-life community sample. Consistent with evidence that negative dispositions are associated with activation of innate inflammatory pathways (e.g., Appels et al., 2000; Maes et al., 1992, 1995; Suarez et al., 2003, 2004) and predict vulnerability to inflammatory disease (for review, see Everson-Rose and Lewis, 2005; Miller et al., 1996), our findings show that individuals who describe themselves as more verbally and physically aggressive have higher levels of plasma CRP and IL-6 than their less antagonistic counterparts. These associations are depicted in Fig. 1, which shows a positive linear relationship between hostile behavioral tendencies and both markers of systemic inflammation. These relationships were independent of demographic and behavioral health risk factors, including age, sex, race, years of education, BMI, hypertension, osteoarthritis, hormone use, smoking, physical activity, sleep volume, and alcohol use, and trait NA. Overall, our findings are consistent with published evidence that dispositional anger/hostility covaries positively with levels of CRP and IL-6 (Coccaro, 2006; Graham et al., 2006; Ranjit et al., 2007; Sjogren et al., 2006; Suarez, 2004) and suggest that hostile behavioral tendencies may confer much of the variability in inflammation associated with negative emotions. 
The current findings also show that cognitive (cynicism and hostile attributions) and affective (anger and irritability) dimensions of hostility, and trait NA are positively associated with plasma levels of IL-6 and CRP independently of demographic and current health factors. These effects were largely explained by correlated variation in BMI and smoking, suggesting that lifestyle parameters provide a pathway linking hostile affect and cognitions to inflammation. In contrast, trait NA did not contribute much that was independent of the affective component of hostility to the prediction of CRP and IL-6. Interestingly, the association between antagonistic behavioral tendencies and inflammation was not explained by lifestyle factors or negative affective style. This raises the possibility that individuals high in aggressiveness are at increased risk of inflammatory disease. In this regard, consistent prospective evidence shows that dispositional anger/hostility predicts incidence and progression of cardiovascular disease (e.g., Chang et al., 2002; Everson et al., 1998; Kawachi et al., 1996; Koskenvuo et al. 1988), which is widely accepted to be of inflammatory etiology (Ridker et al., 2000a, 2000b; Ross, 1999). Further research is warranted to determine if it is the behavioral component of these antagonistic dispositions that confers increased susceptibility to disease.

The current findings may help to shed light on inconsistent findings in the extant literature. To date, studies that have not shown a main effect of hostility on inflammatory mediators have used the full Ho scale, which includes 50 items focusing on multiple dimensions of hostility (Suarez, 2003; Graham et al., 2006; Miller et al., 2003). In contrast, studies finding a positive association between hostility and IL-6 have employed subscales of the Ho that focus on the cognitive or affective dimensions of hostility (e.g., Ranjit et al., 2007). There is also some support in the literature for an interaction between total hostility and depressive symptoms in the prediction of systemic inflammation; however, findings are inconsistent with Ho positively related to IL-6 among individuals higher in depressive symptoms in one study (Suarez et al., 2003) and among those lower in depressive symptoms in another study (Miller et al., 2003). In contrast, in the current study we found no significant interactions between dimensions of hostility and trait NA in the prediction of IL-6 or CRP. Our findings suggest that stronger and more consistent associations may be found using measures that focus on the behavioral dimension of hostility. In support of this possibility, Coccaro (2006) examined a sample of personality disordered subjects at high risk for aggression and hostility and showed a stronger positive association of CRP with trait levels of physical aggression than with more cognitive components of hostility, as defined by the Buss-Durkee Hostility Inventory (Buss and Durkee, 1957).

Numerous behavioral and biological mechanisms have been proposed as potential links between dispositional characteristics and inflammatory processes. Consistent with the extant literature (e.g., Everson et al., 1997; Kawachi et al., 1996), our data show relationships between all dimensions of trait hostility and poorer health practices, including more smoking and alcohol use, higher BMI, fewer hours of sleep/week, and less physical activity. Also consistent with prior reports (e.g., Bermudez et al., 2002; Bruunsgaard, 2005; Frohlich et al, 2003), we found that these lifestyle-related risk factors (higher BMI, smoking, less physical activity and sleep volume) covaried positively with systemic inflammation. As expected, this was particularly the case for BMI, which is likely explained by the peripheral production and release of IL-6 by adipocytes (Berg and Scherer, 2005; Mohamed-Ali et al., 1997) and the IL-6-stimulated production of CRP by hepatocytes (Heinrich et al., 1990). In the current findings, the influences of affective and cognitive dimensions of hostility on systemic inflammation were explained by behavioral factors, including BMI, smoking status and physical activity. Thus, behavioral covariates of hostile affect may provide a pathway to increased systemic inflammation. In contrast, observed relationships between trait differences in aggressive tendencies and lifestyle risk factors on systemic inflammation were largely independent, suggesting that BMI, smoking, alcohol use, hours of sleep, and physical activity do not account for a large portion of the interindividual variability in aggression-related immune function.

Other mechanisms that could underlie associations between trait characteristics and systemic inflammation include biological processes. In this regard, it is widely suggested that antagonistic dispositions are associated with activation of multiple physiological pathways that influence immune function. For example, when compared with less antagonistic individuals, hostile and angry individuals show greater activation of the sympathetic nervous system in response to acute mental challenge (e.g., al'Absi and Bongard, 2006;Suls and Wan, 1993), diminished parasympathetic control over cardiac function (Sloan et al., 1994), and higher circulating concentrations of catecholamines (Suarez et al., 1998) and cortisol (Pope and Smith, 1991). Furthermore, individual differences in the balance of activation of the sympathetic and parasympathetic branches of the autonomic nervous system and in the regulation of the hypothalamic-pituitary-adrenal axis have been associated with modulation of the production of proinflammatory cytokines (Marsland et al., 2007; Suarez et al., 1998), of levels of systemic inflammation (Elenkov and Chrousos, 2002; Miller et al., 2002; Sondergaard et al., 2000), and of susceptibility to health problems associated with inflammatory processes (Chrousos, 1995; Gianaros et al., 2005).

There are a number of limitations of the current study that should be considered in interpreting findings. First, its cross-sectional design precludes causal interpretation. Alternative explanations for our results include reverse causality, with higher levels of systemic inflammation impacting the central nervous system. Indeed, a growing literature supports immune-to-brain communication, with activation of peripheral inflammatory processes signaling the brain and resulting in the so called "sickness syn- 
drome", which includes irritability (Maier and Watkins, 1998). Thus, pathways exist between the immune system and brain regions involved in the regulation of affect, raising the possibility that systemic inflammation results in increased antagonistic behavior. Alternatively, inflammatory mediators and dispositional aggression may be related to a third factor, such as premorbid disease, general health or genetic predisposition. In this regard, our sample was limited by the inclusion of a number of individuals $(12.7 \%)$ who reported some osteoarthritis. We included this as a covariate in all models, so it is unlikely to explain observed associations; however, it raises the challenge of identifying a population clear of any inflammatory disease in the study age range. Finally, recent evidence shows that systemic levels of CRP are highly heritable (Wessel et al., 2007), raising the possibility that genetic factors could contribute to both inflammatory markers and personality.

A second limitation is the use of a single measurement of inflammatory mediators. Although there is evidence that IL-6 and CRP are relatively stable among healthy individuals over extended periods (e.g., Rao et al., 1994), multiple assessments over time would be expected to provide a more reliable measure of individual differences. Finally, the health significance of systemic levels of proinflammatory mediators is difficult to determine, as it is regulation of levels in response to a specific demand that confers immune efficiency. In the future, longitudinal investigations are indicated beginning in early adulthood and tracking the influence of stable individual differences in the dimensions of hostility on inflammatory mediators to better elucidate how personality may shape the health of individuals.

\section{Acknowledgments}

This study was supported by Grants P01HL40962 from the National Heart Lung and Blood Institute (SBM), NR008237 from the National Institute of Nursing Research (ALM) and the John D. and Catherine T. MacArthur Foundation Network of Socioeconomic Status and Health. The expert technical assistance of Ramasri Sathanoori, MS and Cyndi Kravitz is gratefully acknowledged.

\section{References}

Al'Absi, M., Bongard, S., 2006. Neuroendocrine and behavioral mechanisms mediating the relationship between anger expression and cardiovascular risk: Assessment considerations and improvements. J. Behav. Med. 29, 573-590.

Appels, A., Bar, F.W., Bar, J., Bruggeman, C., de Baets, M., 2000. Inflammation, depressive symptomatology, and coronary artery disease. Psychosom. Med. 62, 601-605.

Barefoot, J.C., 1997. Depression and coronary heart disease. Cardiologia 42, 1245-1250.

Barefoot, J.C., Larsen, S., von der Lieth, L., Schroll, M., 1995. Hostility, incidence of acute myocardial infarction, and mortality in a sample of older Danish men and women. Am. J. Epidemiol. 142, 477-484.

Berg, A.H., Scherer, P.E., 2005. Adipose tissue, inflammation, and cardiovascular disease. Circ. Res. 96, 939-949.
Bermudez, E.A., Rifai, N., Buring, J., Manson, J.E., Ridker, P.M., 2002. Interrelationships among circulating interleukin-6, C-reactive protein, and traditional cardiovascular risk factors in women. Arterioscler. Thromb. Vasc. Biol. 22, 1668-1673.

Black, P.H., 2003. The inflammatory response is an integral part of the stress response: Implications for atherosclerosis, insulin resistance, type II diabetes and metabolic syndrome X. Brain Behav. Immun. 17, $350-364$

Boyle, S.H., Jackson, W.G., Suarez, E.C., 2007. Hostility, anger, and depression predict increases in $\mathrm{C} 3$ over a 10-year period. Brain Behav. Immun. 21, 816-823.

Bruunsgaard, H., 2005. Physical activity and modulation of systemic lowlevel inflammation. J. Leukoc. Biol. 78, 819-835.

Buss, A.H., Durkee, A., 1957. An inventory for assessing different kinds of hostility. J. Consult. Psychol. 21, 343-348.

Buss, A.H., Perry, M., 1992. The aggression Questionnaire. J. Pers. Soc. Psychol. 63, 452-459.

Chang, P.P., Ford, D.E., Meoni, L.A., Wang, N.Y., Klag, M.J., 2002. Anger in young men and subsequent premature cardiovascular disease: the precursors study. Arch. Int. Med. 162, 901-906.

Chrousos, G.P., 1995. The hypothalamic-pituitary-adrenal axis and immune-mediated inflammation. New Engl. J. Med. 332, 13511362.

Coccaro, E.F., 2006. Association of C-reactive protein elevation with trait aggression and hostility in personality disordered subjects: a pilot study. J. Psychiat. Res. 40, 460-465.

Contrada, R.J., Jussim, L., 1992. What does the Cook-Medley hostility scale measure? In search of an adequate measurement model. J. Appl. Psychol. 22, 615-627.

Cook, W.W., Medley, D.M., 1954. Proposed hostility and pharisaic-virtue scales for the MMPI. J. Appl. Psychol. 38, 414-418.

Costa, P.T., McCrae, R.R., 1985. Hypochondriasis, neuroticism, and aging: when are somatic complaints unfounded? Am. Psychol. 40, 1928.

Elenkov, I.J., Chrousos, G.P., 2002. Stress hormones, proinflammatory and anti-inflammatory cytokines, and autoimmunity. Ann. N.Y. Acad. Sci. 966, 290-303.

Everson, S.A., Kauhanen, J., Kaplan, G.A., Goldberg, D.E., Julkunen, J., Tuomilehto, J., Salonen, J.T., 1997. Hostility and increased risk of mortality and acute myocardial infarction: the mediating role of behavioral risk factors. Am. J. Epidemiol. 146, 142-152.

Everson, S.A., Goldberg, D.E., Kaplan, G.A., Julkunen, J., Salonen, J.T., 1998. Anger expression and incident hypertension. Psychosom. Med. 60, 730-735.

Everson-Rose, S.A., Lewis, T.T., 2005. Psychosocial factors and cardiovascular diseases. Annu. Rev. Pub. Health 26, 469-500.

Ferrucci, L., Harris, T.B., Guralnik, J.M., Tracy, R.P., Corti, M.C., Cohen, H.J., Penninx, B., Pahor, M., Wallace, R., Havlik, R.J., 1999. Serum IL-6 level and the development of disability in older persons. J. Am. Geriatr. Soc. 47, 639-646.

Frohlich, M., Sund, M., Lowel, H., Imhof, A., Hoffmeister, A., Koenig, W., 2003. Independent association of various smoking characteristics with markers of systemic inflammation in men. Results from a representative sample of the general population (MONICA Augsburg Survey 1994/95). Eur. Heart J. 24, 1365-1372.

Gianaros, P.J., Salomon, K., Zhou, F., Owens, J.F., Edmundowicz, D., Kuller, L.H., Matthews, K.A., 2005. A greater reduction in highfrequency heart rate variability to a psychological stressor is associated with subclinical coronary and aortic calcification in postmenopausal women. Psychosom. Med. 67, 553-560.

Graham, J.E., Robles, T.F., Kiecolt-Glaser, J.K., Malarkey, W.B., Bissell, M.G., Glaser, R., 2006. Hostility and pain are related to inflammation in older adults. Brain Behav. Immun. 20, 389-400.

Heinrich, P.C., Castell, J.V., Andus, T., 1990. Interleukin-6 and the acute phase response. Biochem. J. 265, 621-636.

Kawachi, I., Sparrow, D., Spiro 3rd, A., Vokonas, P., Weiss, S.T., 1996. A prospective study of anger and coronary heart disease. The Normative Aging Study. Circulation 94, 2090-2095. 
Khaodhiar, L., Ling, P.R., Blackburn, G.L., Bistrian, B.R., 2004. Serum levels of interleukin- 6 and C-reactive protein correlate with body mass index across the broad range of obesity. J. Parenter. Enteral. Nutr. 28, $410-415$.

Kiecolt-Glaser, J.K., Glaser, R., 1988. Methodological issues in behavioral immunology research with humans. Brain Behav. Immun. 2, 6778.

Koskenvuo, M., Kaprio, J., Rose, R.J., Kesaniemi, A., Sarna, S., Heikkila, K., Langinvainio, H., 1988. Hostility as a risk factor for mortality and ischemic heart disease in men. Psychosom. Med. 50, 330-340.

Libby, P., Ridker, P.M., 1999. Novel inflammatory markers of coronary risk: theory versus practice. Circulation 100, 1148-1150.

Maes, M., Van der Planken, M., Stevens, W.J., Peeters, D., DeClerck, L.S., Bridts, C.H., Schotte, C., Cosyns, P., 1992. Leukocytosis, monocytosis and neutrophilia: Hallmarks of severe depression. J. Psychiat. Res. 26, 125-134.

Maes, M., Meltzer, H.Y., Bosmans, E., Bergmans, R., Vandoolaeghe, E., Ranjan, R., Desnyder, R., 1995. Increased plasma concentrations of interleukin-6, soluble interleukin-6, soluble interleukin-2 and transferrin receptor in major depression. J. Affect. Disorders 34, 301-309.

Maier, S.F., Watkins, L.R., 1998. Cytokines for psychologists: implications of bidirectional immune-to-brain communication for understanding behavior, mood, and cognition. Psychol. Rev. 105, 83-107.

Marsland, A.L., Gianaros, P.J., Prather, A.A., Jennings, J.R., Neumann, S.A., Manuck, S.B., 2007. Stimulated production of proinflammatory cytokines covaries inversely with heart rate variability. Psychosom. Med. 69, 709-716.

Miller, G.E., Cohen, S., Ritchey, A.K., 2002. Chronic Psychological stress and the regulation of pro-inflammatory cytokines: a glucocorticoidresistance model. Health Psychol. 21, 531-541.

Miller, G.E., Freedland, K.E., Carney, R.M., Stetler, C.A., Banks, W.A., 2003. Cynical hostility, depressive symptoms, and the expression of inflammatory risk markers for coronary heart disease. J. Behav. Med. 26, 501-515.

Miller, T.Q., Smith, T.W., Turner, C.W., Guijarro, M.L., Hallet, A.J., 1996. A meta-analytic review of research on hostility and physical health. Psychol. Bull. 119, 322-438.

Mohamed-Ali, V., Goodrick, S., Rawesh, A., Katz, D.R., Miles, J.M., Yudkin, J.S., Klein, S., Coppack, S.W., 1997. Subcutaneous adipose tissue releases interleukin-6, but not tumor necrosis factor-alpha, in vivo. J. Clin. Endocrinol. Metab. 82, 4196-4200.

Paffenbarger Jr., R.S., Blair, S.N., Lee, I., Hyde, R.T., 1993. Measurement of physical activity to assess health effects in free-living populations. Med. Sci. Sport Exer. 25, 60-70.

Pope, M.K., Smith, T.W., 1991. Cortisol excretion in high and low cynically hostile men. Psychosom. Med. 53, 386-392.

Pradhan, A.D., Manson, J.E., Rifai, N., Buring, J.E., Ridker, P.M., 2001. C-reactive protein, interleukin-6, and risk of developing type 2 diabetes mellitus. JAMA 286, 327-334.

Pradhan, A.D., Manson, J.E., Rossouw, J.E., Siscovick, D.S., Mouton, C.P., Rifai, N., Wallace, R.B., Jackson, R.D., Pettinger, M.B., Ridker, P.M., 2002. Inflammatory biomarkers, hormone replacement therapy, and incident coronary heart disease: prospective analysis from the Women's Health Initiative observational study. JAMA 288, 980-987.

Ranjit, N., Diez-Roux, A.V., Shea, S., Cushman, M., Seeman, T., Jackson, S.A., Ni, H., 2007. Psychosocial factors and inflammation in the multi-ethnic study of atherosclerosis. Arch. Int. Med. 167, 174 181.

Ridker, P.M., Rifai, N., Stampfer, M.J., Hennekens, C.H., 2000a. Plasma concentration of interleukin-6 and the risk of future myocardial infection among apparently healthy men. Circulation 342, 836-843.
Ridker, P.M., Hennekens, C.H., Buring, J.E., Rifai, N., 2000b. C-reactive protein and other markers of inflammation in the prediction of cardiovascular disease in women. New Engl. J. Med. 342, 836-843.

Rao, K.M., Pieper, C.S., Currie, M.S., Cohen, H.J., 1994. Variability of plasma IL-6 and crosslinked fibrin dimers over time in community dwelling elderly subjects. Am. J. Clin. Pathol. 102, 802-805.

Ross, R., 1999. Atherosclerosis - an inflammatory disease. New Engl. J. Med. 340, 115-126.

Sjogren, E., Leanderson, P., Kristenson, M., Ernerudh, J., 2006. Interleukin-6 levels in relation to psychosocial factors: Studies on serum, saliva, and in vitro production by blood mononuclear cells. Brain Behav. Immun. 20, 270-278.

Smith, T.W., Frohm, K.D., 1985. What's so unhealthy about hostility? Construct validity and psychosocial correlates of the Cook and Medley Ho scale. Health Psychol. 4, 503-520.

Sloan, R.P., Shapiro, P.A., Bigger Jr., J.T., Bagiella, E., Steinman, R.C., Gorman, J.M., 1994. Cardiac autonomic control and hostility in healthy subjects. Am. J. Cardiol. 74, 298-300.

Sondergaard, S.R., Ostrowski, K., Ullum, H., Pedersen, B.K., 2000. Changes in plasma concentrations of interleukin- 6 and interleukin-1 receptor antagonists in response to adrenaline infusion in humans. Eur. J. Appl. Physiol. 83, 95-98.

Suarez, E.C., 2004. C-reactive protein is associated with psychologic risk factors for cardiovascular disease in apparently healthy adults. Psychosom. Med. 66, 684-691.

Suarez, E.C., Sherwood, A., Hinderliter, A.L., 1998. Hostility and adrenergic receptor responsiveness: evidence of reduced (beta)-receptor responsiveness in high hostile men. J. Psychosom. Res. 44, 261-267.

Suarez, E.C., 2003. Joint effect of hostility and severity of depressive symptoms on plasma interleukin-6 concentration. Psychosom. Med. 65, 523-527.

Suarez, E.C., Krishnan, R.R., Lewis, J.G., 2003. The relation of severity of depressive symptoms to monocyte-associated proinflammatory cytokines and chemokines in apparently healthy men. Psychosom. Med. 65, 362-369.

Suarez, E.C., Lewis, J.G., Krishnan, R.R., Young, K.H., 2004. Enhanced expression of cytokines and chemokines by blood monocytes to in vitro lipopolysaccharide stimulation are associated with hostility and severity of depressive symptoms in healthy women. Psychoneuroendocrinology 29, 1119-1128.

Suls, J., Wan, C.K., 1993. The relationship between trait hostility and cardiovascular reactivity: a quantitative review and analysis. Psychophysiology 30, 615-626.

Suls, J., Bunde, J., 2005. Anger, anxiety, and depression as risk factors for cardiovascular disease: the problems and implications of overlapping affective dispositions. Psychol. Bull. 131, 260-300.

Tellegen, A., 1982. Brief Manual for the Differential Personality Questionnaire. Unpublished manuscript. University of Minnesota, Minneapolis.

Watson, D., 2000. Mood and temperament. In: Salovey, P. (Ed.), Emotions and Social Behavior. The Guilford Press, New York, USA.

Watson, D., Tellegen, A., 1985. Toward a consensual structure of mood. Psychol. Bull. 98, 219-235.

Wessel, J., Moratorio, G., Rao, F., Mahata, M., Zhang, L., Greene, W., Rana, B.K., Kennedy, B.P., Khandrika, S., Huang, P., Lillie, E.O., Shih, P.A., Smith, D.W., Wen, G., Hamilton, B.A., Ziegler, M.G., Witztum, J.L., Schork, N.J., Schmid-Schonbein, G.W., O'Connor, D.T., 2007. C-reactive protein, an 'intermediate phenotype' for inflammation: human twin studies reveal heritability, association with blood pressure and the metabolic syndrome, and the influence of common polymorphism at catecholaminergic/beta-adrenergic pathway loci. J. Hyperten. 25, 329-343. 